\title{
Zarya Energy Balance Analysis: The Effect of Spacecraft Shadowing on Solar Array Performance
}

David J. Hoffman

Glenn Research Center, Cleveland, Ohio

Vladimir Kolosov

Khrunichev State Research and Production Space Center, Moscow, Russia

Prepared for the

34th Intersociety Energy Conversion Engineering Conference sponsored by the Society of Automotive Engineers

Vancouver, British Columbia, Canada, August 1-5, 1999

National Aeronautics and

Space Administration

Glenn Research Center 


\section{Acknowledgments}

The authors are eager to acknowledge the efforts of Jeff Hojnicki, James Fincannon and Bob Green of NASA Glenn Research Center who are responsible for developing the Zarya (a.k.a. FGB) shadowing analysis model, based on Khrunichev State Research and Production Space Center descriptions, incorporated in GRC's System Power Analysis for Capability Evaluation (SPACE) FORTRAN model of the International Space Station electrical power system.

Available from

NASA Center for Aerospace Information

7121 Standard Drive

Hanover, MD 21076

Price Code: A03
National Technical Information Service 5285 Port Royal Road Springfield, VA 22100 Price Code: A03 


\title{
Zarya Energy Balance Analysis: The Effect of Spacecraft Shadowing on Solar Array Performance
}

\author{
David J. Hoffman \\ NASA Glenn Research Center \\ Cleveland, Ohio 44135 \\ Vladimir Kolosov \\ Khrunichev State Research and Production Space Center \\ Moscow, Russia
}

\begin{abstract}
The first element of the International Space Station (ISS), Zarya, was funded by NASA and built by the Russian aerospace company Khrunichev State Research and Production Space Center (KhSC). NASA Glenn Research Center (GRC) and KhSC collaborated in performing analytical predictions of the on-orbit electrical performance of Zarya's solar arrays. GRC assessed the pointing characteristics of and shadow patterns on Zarya's solar arrays to determine the average solar energy incident on the arrays. KhSC used the incident energy results to determine Zarya's electrical power generation capability and orbit-average power balance. The power balance analysis was performed over a range of solar beta angles and vehicle operational conditions. This analysis enabled identification of problems that could impact the power balance for specific flights during iSS assembly and was also used as the primary means of verifying that Zarya complied with electrical power requirements. Analytical results are presented for select stages in the ISS assembly sequence along with a discussion of the impact of shadowing on the electrical performance of Zarya's solar arrays.
\end{abstract}

\section{INTRODUCTION}

Zarya, meaning "sunrise" in Russian, is the first element of the International Space Station (ISS) and was successfully launched on November 11, 1998. Zarya provides orientation control, communications and electrical power during the early stages of the ISS assembly sequence. Zarya was funded by NASA and built by the Russian aerospace company Khrunichev State Research and Production Space Center (KhSC) in Moscow. As part of the ISS vehicle integrated performance assessments carried out by the program office at NASA Johnson Space Center (JSC), NASA Glenn Research Center (GRC) and KhSC collaborated in performing analytical predictions of the on-orbit electrical performance on Zarya's solar arrays. The assessment consisted of an analysis performed by
GRC to assess the pointing characteristics of and shadow patterns on Zarya's solar arrays. KhSC used the results to determine Zarya's electrical power generation and orbit-average power balance. These results were determined over a range of solar beta angles and vehicle operational conditions and orientations and lead to the identification of conditions that would ensure a positive energy balance. The analysis enabled identification of problems that could impact the power balance for specific flights throughout ISS assembly so that design and/or operational changes could be implemented at an early stage. This analysis was also used as the primary means of verifying that Zarya complied with electrical power requirements.

At present, the ISS configuration consists of the Russian-provided Zarya and the US-provided Node 1 connecting module. Node 1 has been named Unity. Electrical power is generated by the FGB solar arrays and is used to power the FGB itself as well as systems in the Node via a Russian-to-American Converter Unit, or RACU.

\section{SOLAR ARRAY POINTING CONTROL SYSTEM}

Although Zarya is the more colorful name for the first ISS element, engineers in the ISS program refer to Zarya by its original technical name, the Functional Energy Block, and its Russian acronym, FGB. Throughout the remainder of this paper, the Russian acronym is used.

The FGB generates power from two solar arrays. The arrays are mounted on the port and starboard sides of the spacecraft (as shown in figure 1) and are referred to as Solar Array II (SA-II) and Solar Array IV (SA-N), respectively. The arrays can articulate in a single axis only, and thus, cannot completely track the sun in most station attitudes. Pointing control is provided either by sun sensors mounted on the FGB body or by a guidance, navigation and control (GN\&C) computer system in the Service Module (SM) ISS element, when it is present. Launch of the SM should occur in late 1999. When the SM GN\&C system drives the pointing 
system, the arrays track the sun to the extent possble given single-axis pointing. When the sun sensors drive the pointing system, it is accomplished via eight sensors which are mounted on the body of the FGB with four sensors on the forward end and four sensors on the aft end of the spacecraft, as indicated in figure 1. Each sensor has a field of view of approximately one octant $\left(1 / 8^{n}\right)$ of the sky. Thus, the combination of eight sensors can deted the sun in nearly any orientation. The exception is if the sun is very near the $\pm Y_{\text {iss }}$ axis of the station. If the sun is within a $20^{\circ}$ cone angle of the $Y_{\text {ss }}$ axis of the station, it is out of the field-of-view of all the sensors. In this situation, not much power is produced anyway since the solarvector is paralel to the FGB solararray axis.

Whenever any sensor detects the sun, the control system determines the location of the sun relative to one of 16 "zones", each of which is $22.5^{\circ}$ wide. The control system then commands the solar arrays to rotate to the selected zone and the array rotates to the center of that zone and stops. Since the array stops in the center of the zone and the sun could be in any part of the zone, there is a potential positioning error of up to $\pm 11.25^{\circ}$.

The solar arrays do not rotate continuously through $360^{\circ}$. There is a "stopper" at the end of zone number 16 through which the arrays cannot cross. This stopper is located in the $+Z_{\text {Iss }}$ axis of the station. It is positioned such that in a normal Local Vertical/Local Horizontal (LVLH or +XvV+Znadir) flight mode, the arrays would never need to cross the stopper in order to track the sun. If an array is on one side of the stopper when the control system commands it to a position on the opposite side (e.g. the array is in zone \#16 and is commanded to move to zone \#1), the array will rotate around in the opposite direction in order to reach the commanded zone (e.g. it will move from \#16 to \#15 to \#14 ...).

When commanded to move to a new zone, the solar arrays move at their maximum rate of $42 \%$ minute. The arrays stop such that the array normal is at the center of the zone, within a placement error of $\pm 3^{\circ}$.

However, there are conditions in which none of the sun sensors see the sun. This can happen for a number of reasons including: the spacecraft is in the eclipse portion of the orbit, the sun sensors are shadowed by other parts of the station, or the sun is outside of the field of view of the sensors. Whether the solar arrays are stopped in one of the designated zones, or they are moving between zones, they immediately stop tracking whenever the sun sensors cannot see the sun. The arrays remain in that location until one of the sun sensors reacquires the sun.

\section{GRC SOLAR ARRAY POINTING/SHADOWING MODEL}

NASA GRC has modified its ISS electrical power system FORTRAN model called SPACE to perform FGB solar array pointing and shadowing analysis [1].

The FGB solar arrays are pointed in SPACE in a similar fashion to the way they are pointed by the on-orbit control system. The model steps through the entire orbit

NASA/TM- 1999-209299 twice. The fist time through, all the articulating parts of the station (the US solar arrays, SM arrays, etc.), except the FGB arrays, are positioned as they would be based on their respective control systems. Then the model determines which FGB sun sensor sees the sun at each point in the orbit, and then determines whether or not that sensor is shadowed by some other part of the station. Note that due to the geometry of the FGB, it is impossible for the field-of-view of the FGB sun sensors to be blocked by the FGB arrays, and thus, the position of the FGB arrays is not important in determining if the sun sensors are shadowed.

Once the shadowing of the sun sensors has been analyzed for each time step in the orbit, the model passes through the orbit a second time to determine the pointing of the FGB arrays. An arbitrary assumption is made that the arrays are in zone $\# 9$ at the start of the orbit. Then the model looks at each time step, and if any sensor can see the sun, a commanded zone is calculated and the array is moved toward that zone at the maximum allowable rate. If the commanded zone can be reached within the time step, the array is stopped at the commanded zone. If the zone cannot be reached, it is moved toward the commanded zone at the maximum rate for the entire time step. If no sensor can see the sun, then the array is held in the location that it was at during the previous time step.

This method continues for each time step until the end of the orbit is reached. If a single orbit is being analyzed, then the position of the array at the end of the orbit is compared to the position at the start of the orbit. If these positions differ, then a new initial position is selected and the orbit is reassessed, until the initial and ending positions match. Once the position of the FGB arrays are determined for each time step in the orbit, the code then proceeds to determine the shadowing on the FGB arrays from other station components.

Once the code completes all of these calculations, an output file is generated. This file contains the following information for each timestep:

- The cosine of the angles between the port and starboard solar array normal vectors and the sun vector.

- The percentage of the port and starboard array areas that are shadowed.

- The number of the "active" sun sensor and whether or not the sensor is shadowed.

- The zone number where the port solar array is located.

The code also calculates orbit averages for the following:

- The cosine of the angle between the array normal and the sun vector, for the port and starboard arrays, and the average of the two arrays.

- The incident energy fraction (defined in the Results section) for the port and starboard arrays, and the average of the two arrays.

While the solar array pointing and shadowing results from GRC's SPACE model provides detailed information at one minute intervals throughout an orbit, it is the average incident energy fractions that get passed into KhSC's FGB Power Balance Model. 


\section{KHSC POWER BALANCE MODEL}

KhSC has developed a PC-based model that calculates FGB solar array power generation, based on GRC's incident energy analysis, and ISS power consumption, based on a detailed list of FGB subsystem and Node 1 power requirements. The model determines FGB power balance as a function of ISS orientation and operating mode. Basically, the model calculates the state-of-charge of the FGB's $\mathrm{NiCd}$ chemical storage batteries given the energy available during the daylight or insolation period of the orbit and the depth-of-discharge experienced by the batteries over the entire orbit. The model accounts for the efficiencies of all major elements of the FGB electrical power system (figure 2).

During insolation, the power available from the FGB solar arrays is compared with the power required by the FGB itself and Node 1, accounting for conversion and distribution efficiencies. If the power available is greater than that demanded, the excess power is used to recharge the FGB's NiCd batteries. The model accounts for both complete and incomplete charge modes where the batteries are charged to $100 \%$ and $80 \%$ of their capacity, respectively. The incomplete charge mode is the nominal mode used to conserve battery life. The battery state-of-charge is thus determined at the end of insolation.

During orbit night, discharge of the batteries to meet FGB and Node 1 power demands is modeled accounting for conversion and distribution efficiencies. In some cases, the batteries will discharge during insolation. This occurs when the solar arrays can not meet the power demand themselves or are sufficiently shadowed or offpointed from the sun because the sunsensors are shadowed, or during Space Shuttle Orbiter approach and rendezvous when the FGB solar arrays are feathered in order to avoid rocket exhaust plumes.

In conjunction with the GRC incident energy average analysis, the KhSC power balance analysis has been performed for the initial iSS assembly flight, 1AR, which was the FGB by itself, through flight 12A, when the second of four US PV power modules is deployed and the FGB solar arrays are retracted.

\section{RESULTS}

FGB incident energy average and power balance results are fully described in ISS program documentation [2]. In this paper, results for isS assembly flights/stages $2 \mathrm{~A}, 4 \mathrm{~A}$ and $12 \mathrm{~A}$ are highlighted, illustrating the effects of solar array offpointing and shadowing. Figures 3 through 5 show the ISS configuration for each of these stages. The term "flight" refers to the instances where the Orbiter is present. After the Orbiter has departed, the remaining ISS vehicle is referred to as a "stage".

A key parameter resulting from the GRC analysis, and a required input to the KHSC power balance analysis, is the incident energy fraction defined as

$$
(1 / T \text { sun }) \Sigma\left\{\operatorname{Cos} \theta_{i}\left(100-\text { Shadow }_{i}\right) / 100\right\}
$$

where Tsun is the insolation period duration, $\theta_{i}$ is the angle between the solar vector and the array normal at timestep i, and Shadow is the percentage of solar array surface area being shadowed at timestep i.

Results forboth incident energy fraction and FGB power balance are given as a function of the absolute value of the solar beta angle, $\beta$, which is the angle between the solar vector and the nomal to the orbit plane.

Also, the results are presented for a number of ISS flight orientations. The nominal flight mode for low solar beta angles is "Local Vertical/Local Horizontal (LVLH)" where the ISS + $X$ axis points in the direction of the velocity vector ( $v v$ ) and the ISS $+Z$ axis points in the nadir direction, towards Earth $(+X v v+Z$ nadir on the plots). As the solar $\beta$ increases, there will be cosine losses in LVLH due to the fact that the solar arrays can only track on one axis. Therefore, during high solar beta conditions, the ISS flies in a semi-inertial orientation where the ISS $X$ axis is perpendicular to the orbit plane (Xpop). This mode allows the solar array pointing system to keep the arrays normal to the sun. There are a few orientations for Orbiter approach and docking to the ISS. For flight $2 \mathrm{~A}$, this orientation is +Xzenith-ZvV. For flights $4 A$ and $12 A$, the Orbiter rendezvous when the ISS is in the LVLH, or $+X v v+Z$ nadir orientation. While the Orbiter is docked, the ISS will fly in either LVLH or Xpop orientations depending on the solar beta angle.

\section{FLIGHT/STAGE 2A}

During ISS assembly flight $2 \mathrm{~A}$ in December 1998, the Space Shuttle Orbiter brought Node 1, Unity, to orbit and attached it to the FGB. After the Orbiter's departure, ISS stage $2 \mathrm{~A}$ consisted of the FGB and Node 1.

In addition to the ISS flight modes discussed in the previous section, during stage $2 A$ there is a mode known as "Xnadir Spin". In this mode, the ISS slowly rotates $\left(18^{\circ} /\right.$ minute) about its $+X$ axis which is pointing in the nadir direction, towards Earth. This is done to conserve attitude control fuel.

Incident energy fractions and power balance results for ISS assembly flight 2A (for Orbiter rendezvous and mated configurations) and ISS stage $2 A$ (FGB and Node 1 alone) are given in figures 6 and 7 respectively.

\section{Orbiter Approach}

For this flight, during Orbiter approach and docking, the ISS is in the +Xzenith-Zvv orientation with the FGB's solar arrays feathered, i.e. held at a particular angle in order to minimize Orbiter rocket exhaust plume impingement. In this situation, the front side of the FGB solar arrays are not illuminated past orbit noon. This, in addition to shadowing from the Orbiter, leads to low average incident energy fraction $(0.28$ for low $|\beta|, 0.16$ for high $|\beta|$ ). Since the solar arrays are not tracking the sun and only generate power for a portion of the daylight period, the NiCd batteries are used to maintain a positive power balance. The model does not account for power produced by the back side of the FGB arrays. 


\section{Orbiter Mated}

While the Orbiter is mated to the ISS in the $+X v v$ Znadir orientation, incident energy losses occur due to the inability of the solar arrays to track in two-axes, i.e. $\cos (\beta)$ losses, shadowing of the sun sensors and/or solar arrays, and "rewinding" of solar array gimbal due to "stopper". Additional losses occur when the absolute value of the solar $\beta$ angle is less than $30^{\circ}$ because the Orbiter and Node shadow the sun sensors after orbit dawn. No incident energy losses occur while the Orbiter is mated to the ISS in the Xpop orientation. In figure 7 , the plots show power balance as the difference between power generation and power consumption. Positive power balance for the LVLH configuration occurs when $|\beta|$ is less than $37^{\circ}$. Positive power balance occurs over the full range of solar $\beta$ for the Xpop configuration.

\section{ISS-Alone Operations}

While in the Xnadir spin orientation, the spinning motion causes both pointing losses and shadowing of the arrays by the FGB itself, leading to a fairly constant average incident energy fraction $(0.68$ and 0.72 for low $|\beta|, 0.60$ to 0.33 for high $|\beta|$ ). In the LVLH orientation, the incident energy fraction decreases with the cosine of the solar $\beta$ angle. Additional incident energy losses are due to the front of the array not being illuminated at orbit dawn, the sun sensors being shadowed near orbit dawn when $|\beta|$ is less than $30^{\circ}$, shadowing from the $F G B$ itself and the sun sensor "blind spot" for $|\beta|$ near $70^{\circ}$. Positive power balance for the LVLH occurs when $|\beta|$ is less than $30^{\circ}$ and over the full range of solar $\beta$ for the $X p o p$ configuration.

\section{FLIGHT/STAGE 4A}

On ISS assembly flight 4A planned for early 2000 , the Orbiter will bring the first US PV module to orbit. Called P6 for its ultimate location on the ISS truss, this PV module will be placed in a temporary location on the $\mathrm{Z1}$ Truss. At this point in the assembly sequence, the Service Module is present. As previously discussed, the SM's GN\&C computer can drive the FGB's solar array pointing system. Figures 8 and 9 depict incident energy fractions and power balance results for ISS assembly flight/stage 4A. The power balance results in figure 9 are for the situation where the sun sensors drive the pointing of the FGB arrays.

\section{Orbiter Approach}

For this assembly flight and those after, the ISS is in the LVLH (+Xvv+Znadir) orientation for Orbiter approach and docking. Given this orientation and the solar array feather angles, the FGB solar arrays are not illuminated until after orbit noon leading to low incident energy fractions.

\section{Orbiter Mated}

When the Orbiter is mated to the ISS in the + XvvZnadir orientation and the FGB solar array pointing is being performed by GN\&C system, there

NASATM- 1999-209299 are incident energy losses due to offpointing $(\operatorname{Cos}(\beta))$, and because the FGB solar arrays are eclipsed by $S M$ and P6 arrays once per orbit, and the starboard array is shadowed by Node 1 and the FGB and Orbiter when $|\beta|>30^{\circ}$.

When the FGB solar array pointing is performed by the sun sensors, the same incident energy losses as described above are experienced. Additional incident energy losses occur due to the array front side not being illuminated at orbit dawn, Orbiter and Node 1 shadowing the sun sensors after orbit dawn when $|\beta|$ is less than $30^{\circ}$ and additional sun sensor shadowing after orbit noon. In the Mated Xpop orientation, there is slight shadowing of FGB array by the P6 array when the solar $\beta$ is $0^{\circ}$.

Concerning power balance during the Orbiter mated configuration, positive balance occurs over the entire range of solar $\beta$ for the mated Xpop configuration whether or not power is being transterred to the USOS (Node 1). For mated LVLH, positive power balance occuis only when no power is transferred to the USOS. Using the GN\&C system to point the FGB arrays will improve the margin on the power balance when the absolute value of the solar $\beta$ is less than $30^{\circ}$.

\section{ISS-Alone Operations}

When the ISS is in the Xpop orientation, the FGB arrays are increasingly shadowed by $S M$ arrays for $|\beta|$ greater than $60^{\circ}$ for both array pointing methods (sun sensor and GN\&C).

For the LVLH orientation when FGB solar array pointing is accomplished by the GN\&C system, there are incident energy losses due to offpointing $(\cos (\beta))$, the FGB solar arrays being eclipsed by the $P 6$ and SM arrays once per orbit, and one of the FGB arrays being significantly shadowed by Node 1 and the FGB when $|\beta|$ is greater than $30^{\circ}$.

When there is a power transfer of $1200 \mathrm{~W}$ to Node 1 , positive power balance occurs only during Xpop when $|\beta|$ is less than $70^{\circ}$. When there is no USOS power transfer, positive power balance occurs when $|\beta|$ is less than $52^{\circ}$ in LVLH and over the entire range of solar $\beta$ for Xpop, until perhaps the $\max |\beta|$ of $75^{\circ}$. As for the mated configuration, power balance margins will improve in LVLH when using GN\&C pointing for $|\beta|$ less than $30^{\circ}$.

\section{FLIGHT/STAGE 12A}

The second of four US PV modules will be installed during ISS assembly flight $12 \mathrm{~A}$. At this point, ISS stage 12A will consist of a number of major components including the Russian-built Science Power Platform (SPP) and its associated solar arrays. It is also at this stage that the FGB solar arrays will be retracted given the significant shadowing caused by the US PV arrays, the SPP arrays and other major structures.

Figure 10 gives the incident energy results for this flight/stage. A power balance plot is not given because the US PV modules are providing the majority of the power to US-provided ISS systems. 
The power generated by the FGB arrays is still used in the FGB itself. If necessary, power can be transferred into the $F G B$ via the American-to-Russian converter unit (ARCU).

In all cases, there is significant shadowing of both the FGB sun sensors and solar arrays. Large decreases in incident energy averages are seen even in Xpop, when shadows from the P6 and Science Power Platform (SPP) arrays are cast on the FGB arrays.

\section{CONCLUSION}

Incident energy analysis accounting for solar array pointing and shadowing characteristics is an essential first step in performing ISS electrical power balance analysis. Electrical power balance analysis is critical in determining the success of ISS operations. The NASA Glenn Research Center FGB incident energy analysis in conjunction with the Khrunichev FGB power balance analysis assured the ISS Program Office that sufficient power could be produced in the early stages of what will hopefully be a long and productive International Space Station life.

\section{CONTACT}

David J. Hoffman

NASA Glenn Research Center, 21000 Brookpark Road, MS 500-203, Cleveland, Ohio 44135

Phone: (216)433-2445

Fax: (216)433-2995

Email: dihoffman@grc.nasa.gov

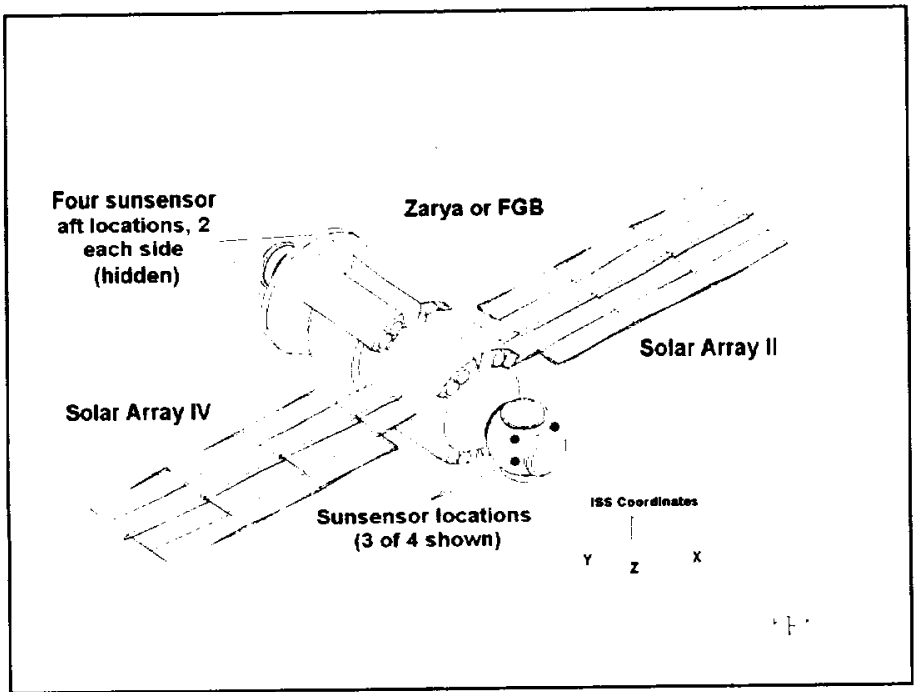

Figure 1 - "Zarya" or FGB sun sensor locations.

\section{REFERENCES}

1- Hojnicki, Jeffrey S., Green, Robert D., Kerslake, Thomas W., McKissock, David B. and Trudell, Jeffrey J., Space Station Freedom Electrical Performance Model, NASA Technical Memorandum 106395, $28^{\text {th }}$ IECEC, Atlanta, Georgia, August 1993.

2- Calculation of Power Balance of FGB Energy Block for ISS Program, Report EN-14B, September 30, 1998, Khrunichev State Research and Production Space Center.

\section{DEFINITIONS, ACRONYMS, ABBREVIATIONS}

ARCU:

American-to-Russian Converter Unit

FGB:

Functional Energy Block or Zarya

GN\&C:

Guidance, Navigation and Control

Incident Energy Fraction:

$(1 /$ sun $) \Sigma\left\{\operatorname{Cos} \theta_{i}\left(100-\right.\right.$ Shadow $\left.\left._{i}\right) / 100\right\}$

iss:

International Space Station

LVLH:

Local Vertical/Local Horizontal (also +XvvZnadir)

PV:

Photovoltaic

RACU:

Russian-to-American Converter Unit

SM:

Service Module

SPP:

Science Power Platform

USOS:

United States Orbital Segment

Xpop:

$\mathrm{X}$-axis Perpendicular to the Orbit Plane

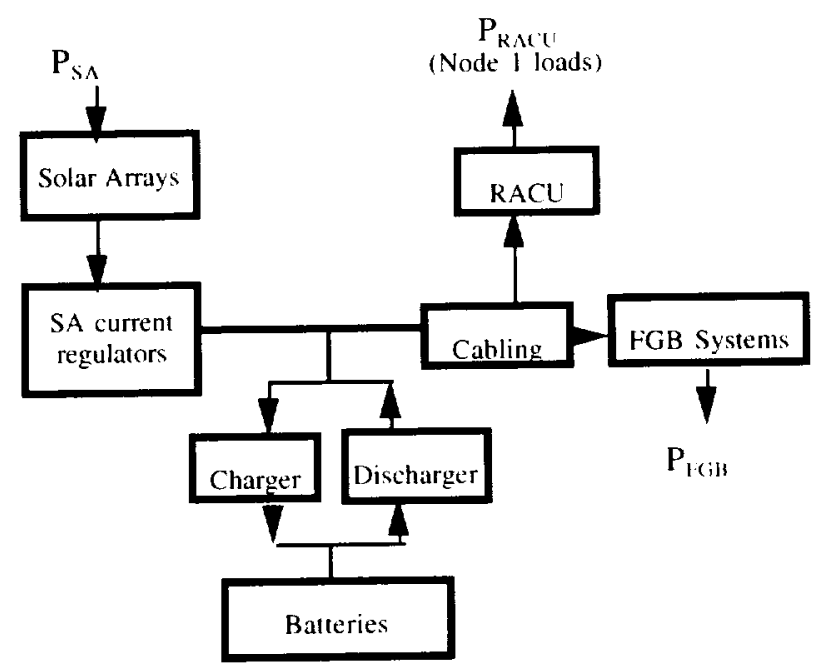

Figure 2 - FGB EPS Block Diagram 

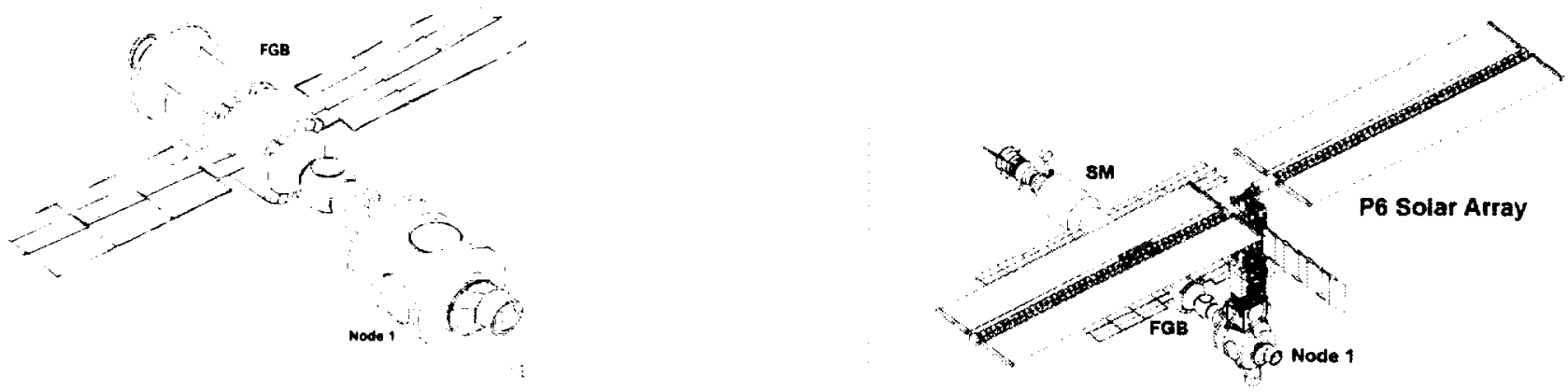

Figure 3 - ISS Stage 2A

Figure 4 - ISS Stage 4A

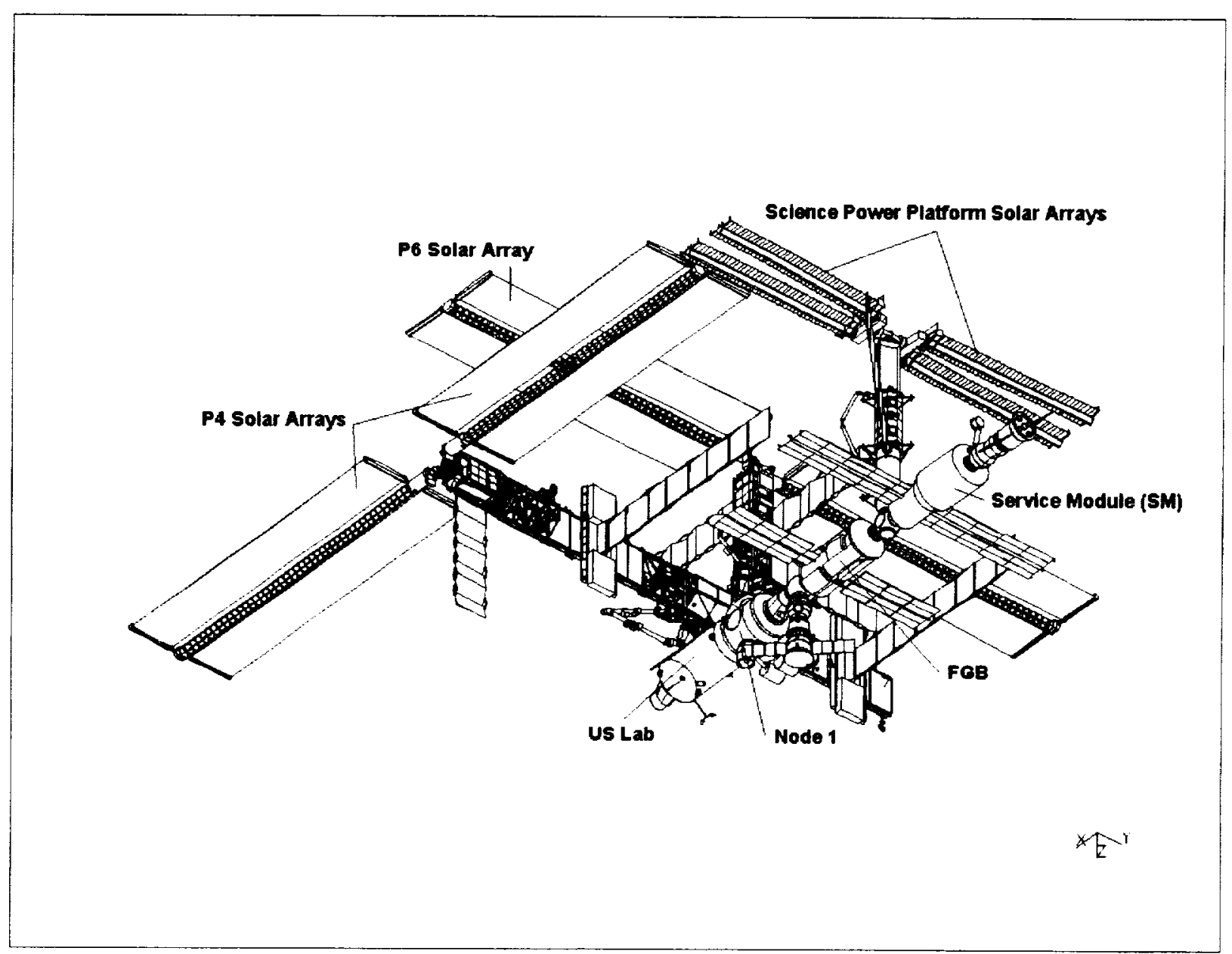

Figure 5 - ISS Stage 12A 


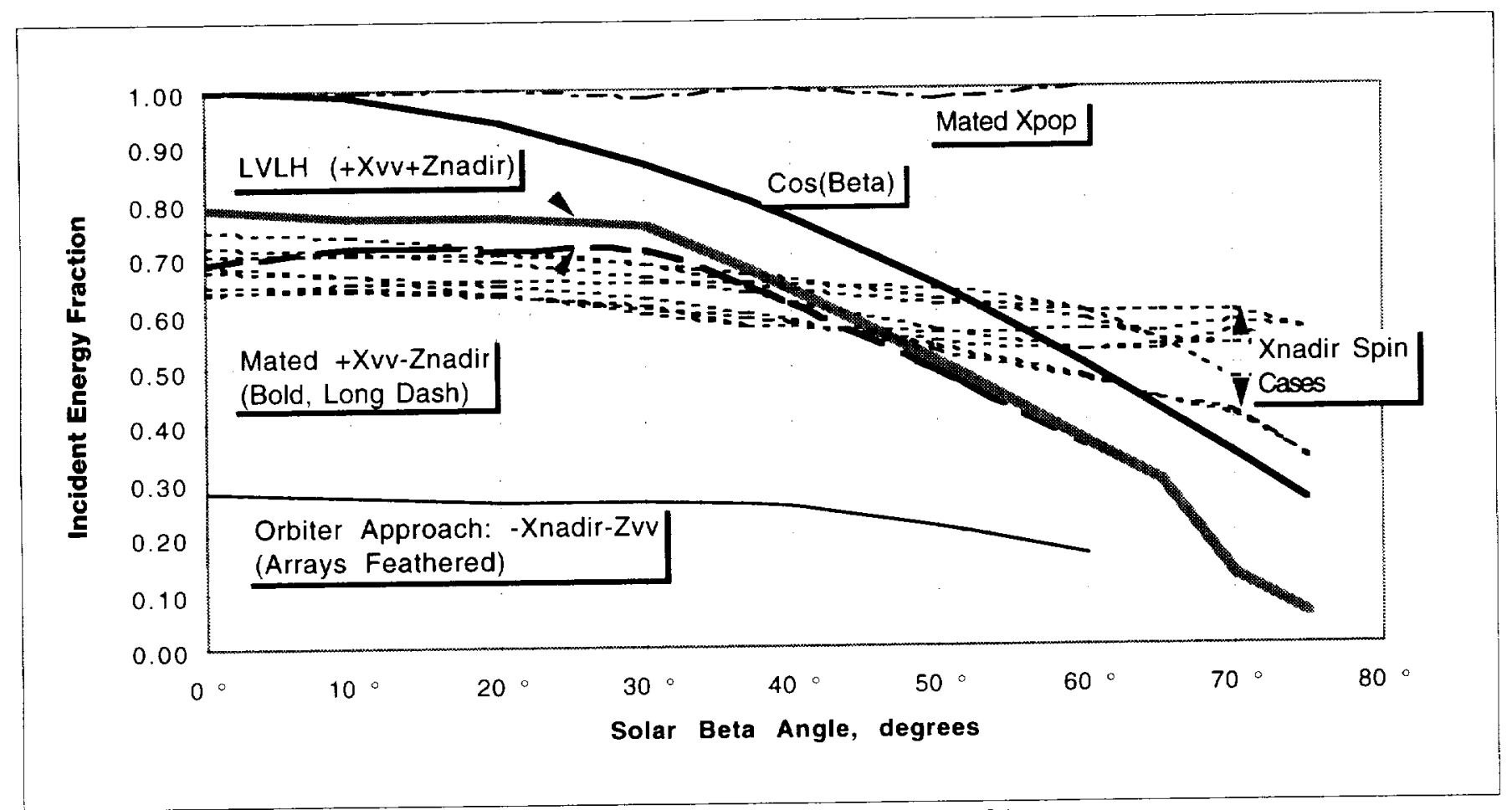

Figure 6 - Incident Energy Flight/Stage 2A

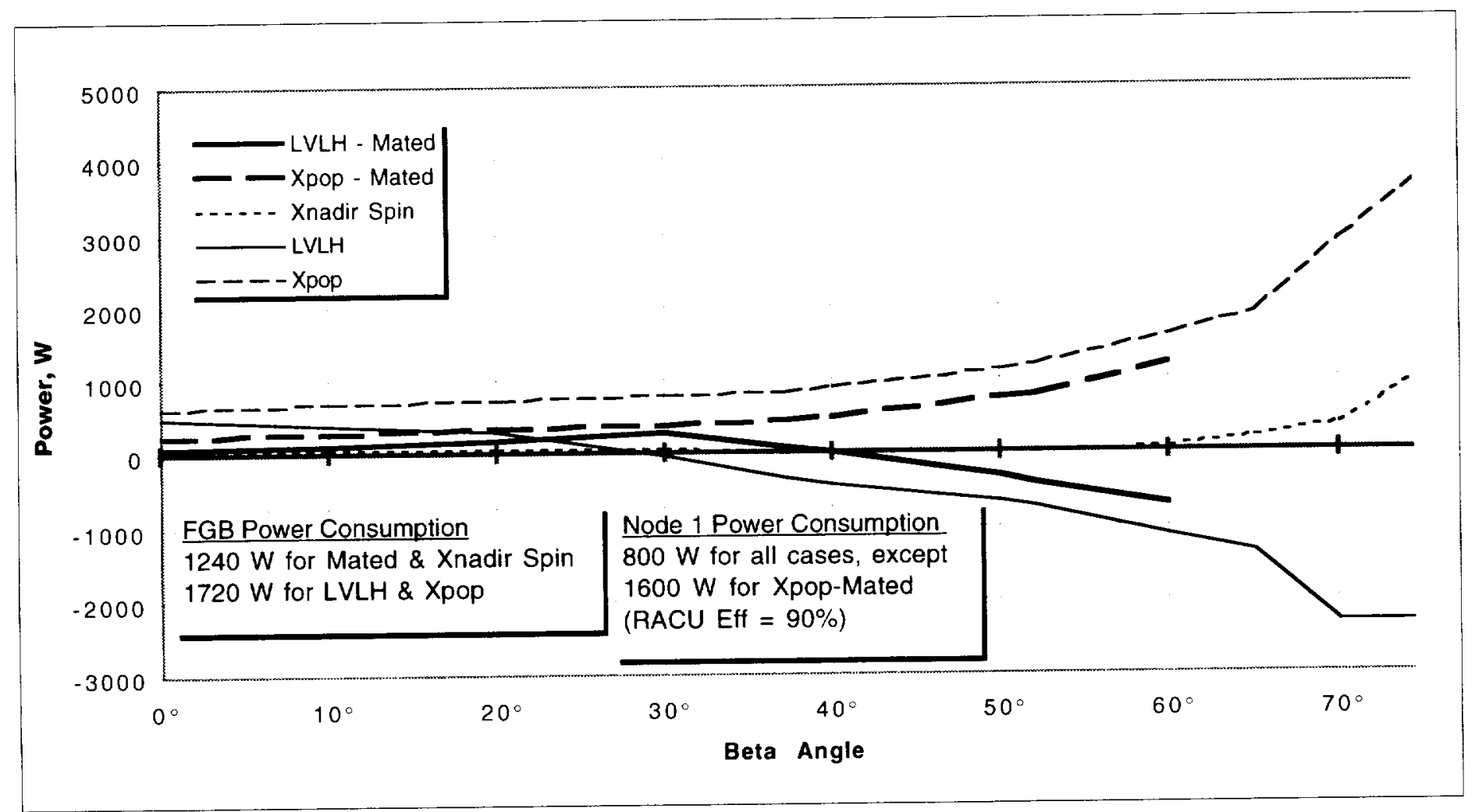

Figure 7 - ISS Power Balance Flight/Stage 2A 


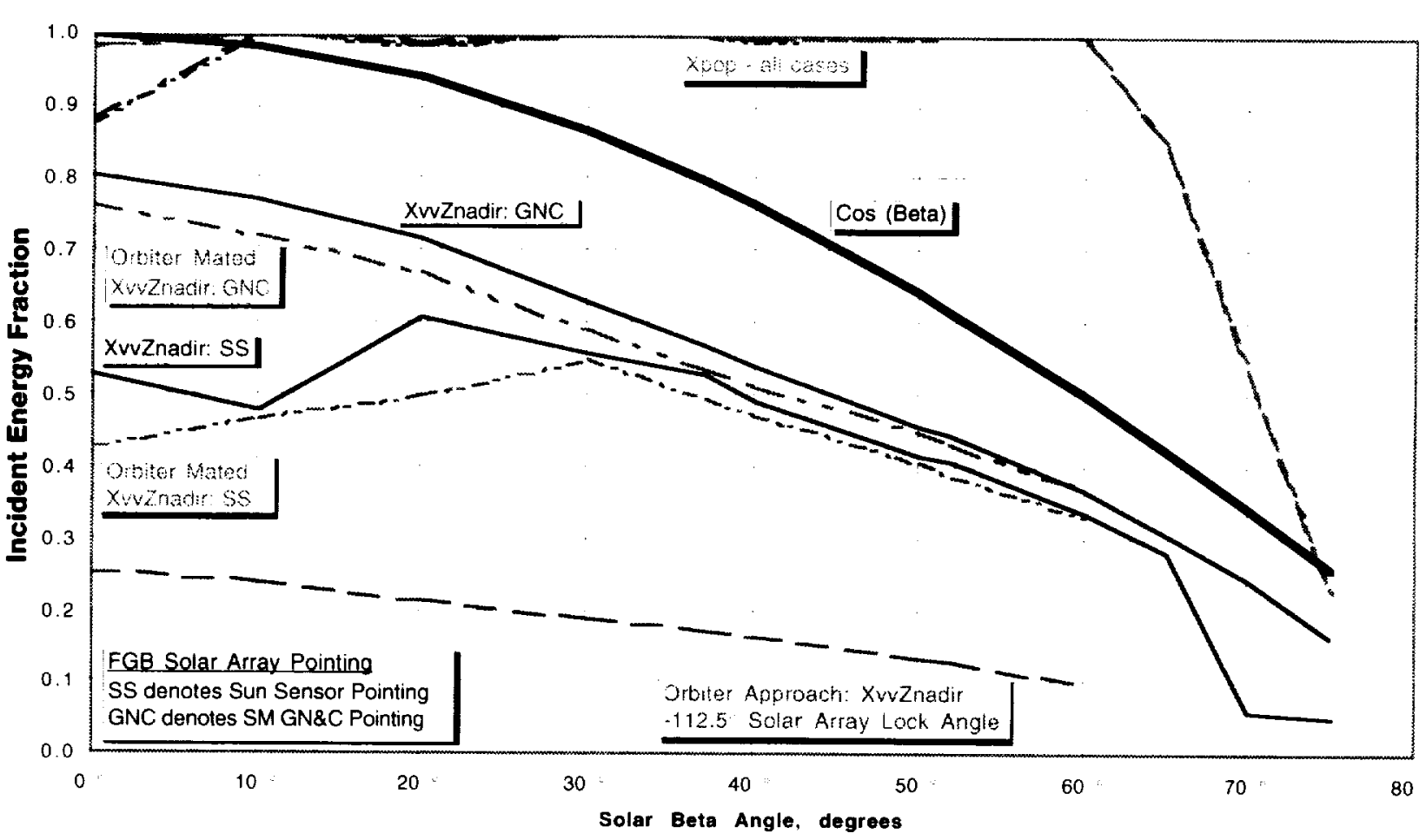

Figure 8 - Incident Energy Flight/Stage 4A

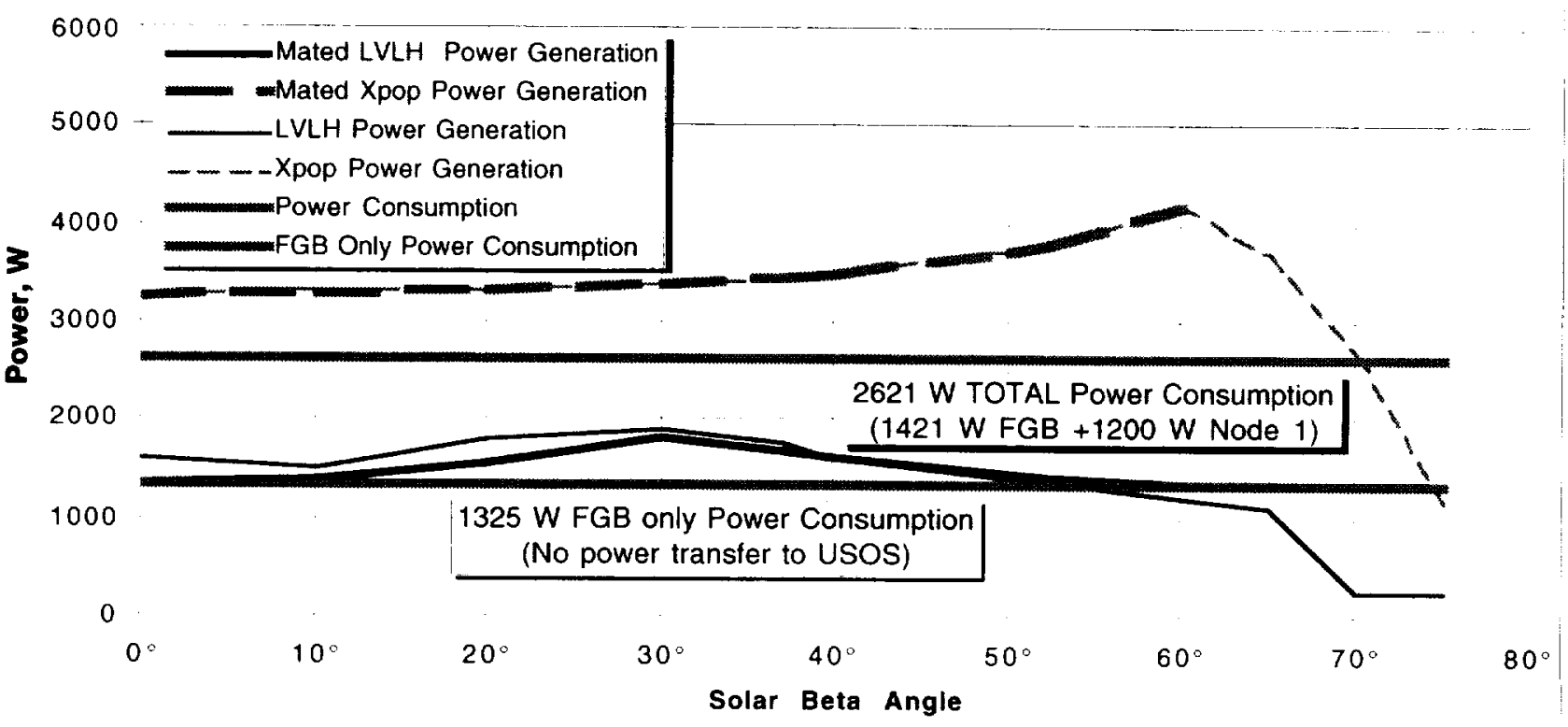

Figure 9 - ISS Power Balance Flight/Stage 4A 


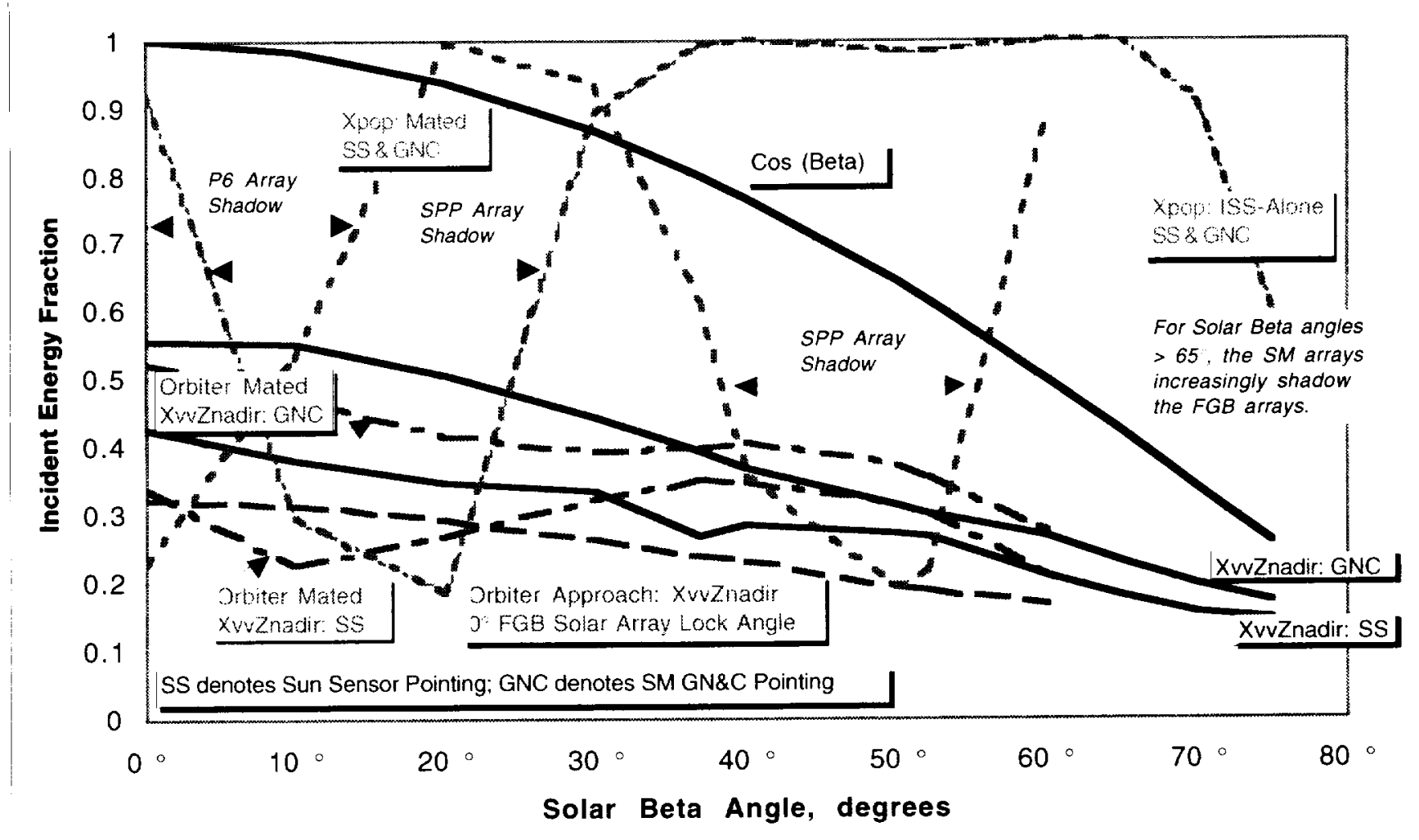

Figure 10 - Incident Energy Flight/Stage 12A 


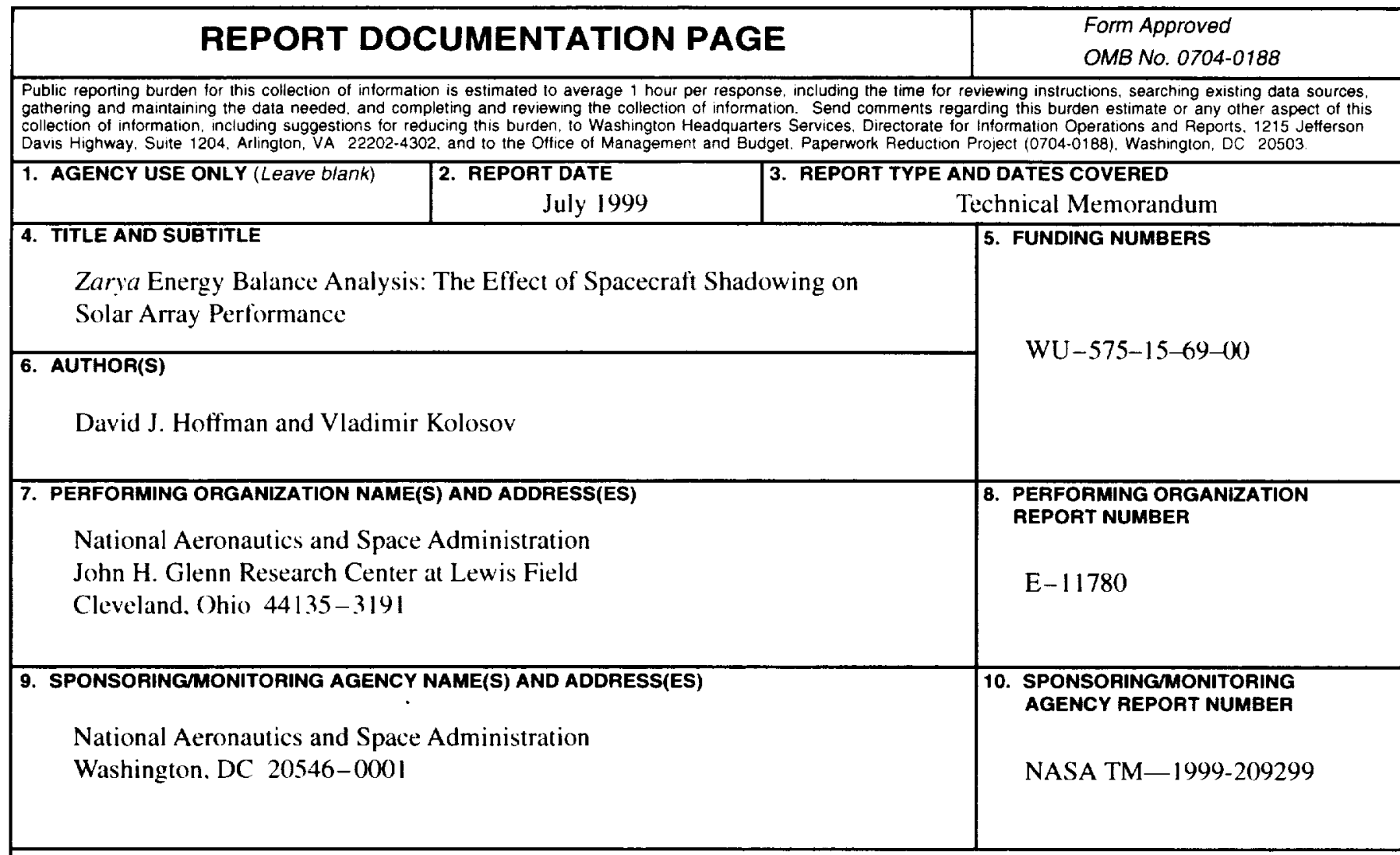

\section{SUPPLEMENTARY NOTES}

Prepared for the 34th Intersociety Energy Conversion Engineering Conference sponsored by the Society of Automotive Engineers, Vancouver, British Columbia, Canada, August 1-5, 1999. David J. Hoffman, NASA Glenn Research Center, and Vladimir Kolosov. Khrunichev State Research and Production Space Center, Moscow, Russia. Responsible person, David J. Hoffman, organization code 6920, (216) 433-2445.

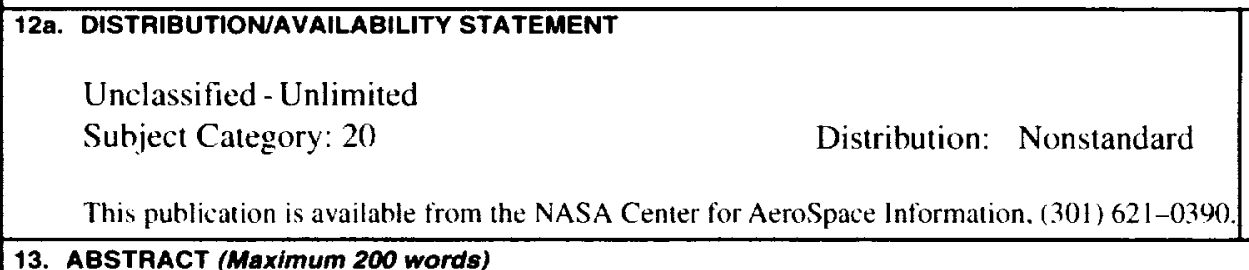

13. ABSTRACT (Maximum 200 words)

The first element of the International Space Station (ISS), Zarya, was funded by NASA and built by the Russian aerospace company Khrunichev State Research and Production Space Center (KhSC). NASA Glenn Research Center (GRC) and KhSC collaborated in performing analytical predictions of the on-orbit electrical performance of Zarya's solar arrays. GRC assessed the pointing characteristics of and shadow patterns on Zarya's solar arrays to determine the average solar energy incident on the arrays. KhSC used the incident energy results to determine Zarya's electrical power generation capability and orbit-average power balance. The power balance analysis was performed over a range of solar beta angles and vehicle operational conditions. This analysis enabled identification of problems that could impact the power balance for specific flights during ISS assembly and was also used as the primary means of verifying that Zarya complied with electrical power requirements. Analytical results are presented for select stages in the ISS assembly sequence along with a discussion of the impact of shadowing on the electrical performance of Zarya's solar arrays.

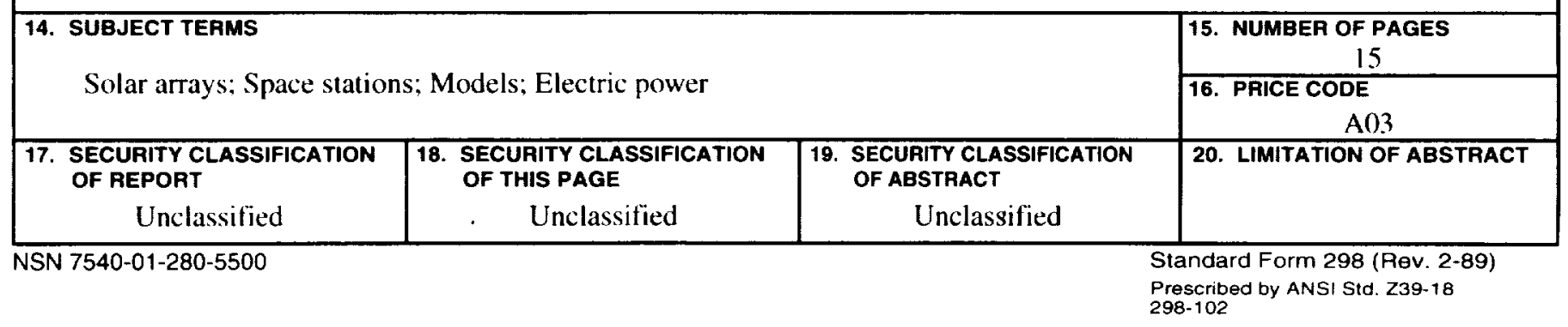

